\title{
Covariant canonical formalism for four-dimensional BF theory
}

\author{
Mauricio Mondragón诂 and Merced Montesinos \\ Departamento de Física, Cinvestav, \\ Av. Instituto Politécnico Nacional 2508, San Pedro Zacatenco, \\ 07360, Gustavo A. Madero, Ciudad de México, México
}

(Dated: September 3, 2018)

\begin{abstract}
The covariant canonical formalism for four-dimensional BF theory is performed. The aim of the paper is to understand in the context of the covariant canonical formalism both the reducibility that some first class constraints have in Dirac's canonical analysis and also the role that topological terms play. The analysis includes also the cases when both a cosmological constant and the second Chern character are added to the pure BF action. In the case of the BF theory supplemented with the second Chern character, the presymplectic 3-form is different to the one of the BF theory in spite of the fact both theories have the same equations of motion while on the space of solutions they both agree to each other. Moreover, the analysis of the degenerate directions shows some differences between diffeomorphisms and internal gauge symmetries.

PACS numbers: 04.20. Fy
\end{abstract}

\footnotetext{
* Current address: Centre de Physique Théorique, Campus de Luminy, F-13288 Marseille, France. Electronic address: mmondrag@cpt.univ-mrs.fr

$¥$ Associate Member of the Abdus Salam International Centre for Theoretical Physics, Trieste, Italy.

$\dagger$ Electronic address: mo@fis.cinvestav.mx

$\S$ Electronic address: merced@fis.cinvestav.mx
} 


\section{INTRODUCTION}

In the path integral quantization of a given field theory one needs to sum the exponential of the classical action weighted with a suitable factor over all possible configurations of the fields under consideration. If the theory under study is a gauge theory one needs, in addition, to factor out the gauge transformations in such a way that the sum includes only equivalence classes of gauge transformed fields. So, intuitively, it is expected that any change in the action principle, yielding the same classical equations of motion, provides a completely different quantum theory. For instance, if the Yang-Mills Lagrangian density $\operatorname{tr} F \wedge \star F$ is modified adding the $\operatorname{term} \theta \operatorname{tr} F \wedge F$ the resulting quantum theory is sensitive to this contribution even when it does not modify the classical equations of motion [1]. One way to understand, at the classical level, the cause of having a different quantum theory for the Yang-Mills field is to realize that, in the generic case, the specification of the Lagrangian density is equivalent to specify the symplectic geometry in the various phase spaces associated with the classical theory. Thus, if the Lagrangian density changes, the symplectic geometry also does generically. If one accepts that what defines a dynamical system is its equations of motion then this knowledge is not enough to specify the symplectic geometry on the various phase spaces involved. If, one the other hand, one accepts that what defines a dynamical system is its equations of motion plus an action principle (which provides its equations of motion), then one is in a different situation. The difference is that, as we have already mentioned, the specification of the Lagrangian density provides a symplectic structure. Thus, an action principle plays a double role: (1) it provides the equations of motion and also (2) provides the symplectic geometry. Before going into the analysis of the BF theory, which is the subject of this paper, let us emphasize this point with a very simple example borrowed from dynamical systems with a finite number of degrees of freedom. The equations of motion for the two-dimensional isotropic harmonic oscillator $\ddot{x}+\omega^{2} x=0$ and $\ddot{y}+\omega^{2} y=0$ can be obtained from the Lagrangian $\mathcal{L}(x, y, \dot{x}, \dot{y})=m\left(\dot{x} \dot{y}-\omega^{2} x y\right)$ or from $\mathcal{L}_{\text {usual }}(x, y, \dot{x}, \dot{y})=\frac{1}{2} m\left(\dot{x}^{2}+\dot{y}^{2}-\omega^{2} x^{2}-\omega^{2} y^{2}\right)$. Moreover, $\mathcal{L} \neq \mathcal{L}_{\text {usual }}+d F(x, y, t) / d t$. Note also that we are not making a change of coordinates, which are the same for both cases. The symplectic structures coming from these Lagrangians are very distinct to each other even when they both provide the same equations of motion [2]. Coming back to field theory, it has been shown, in the context of Dirac's canonical analysis, that the symplectic potential 
changes if one adds topological terms to the Lagrangian density of tetrad gravity [3]. On the other hand, using the covariant canonical formalism, it has been shown that the inclusion of topological terms in Lagrangians for string theory also modifies the original symplectic potential [4, 5].

In this paper, in the context of the covariant canonical formalism [6, 7, 8, 9], we perform the covariant canonical analysis of four-dimensional BF theory, BF theory plus a cosmological constant $\Lambda$, and BF plus the second Chern character $F^{I J} \wedge F_{I J}$. These theories are topological in the sense that there is no fixed background metric $g$ on the four-dimensional manifold $\mathcal{M}$ in which they are defined. In addition, they are topological in the sense that they have no local degrees of freedom. A more detailed analysis of the covariant canonical formalism for BF theory can be found in Ref. [10]. Of course, the inclusion of the second Chern character does not modify the equations of motion while the cosmological constant does. However, the aim of the paper is to study the symplectic geometry involved. It must be emphasized that Dirac's canonical analysis for BF theory has been already done [11, 12] (see also the Appendix A). In Ref. [12] it is shown that the first class constraints $\widetilde{\Psi}_{I J}^{a}:=\frac{1}{2} \widetilde{\eta}^{a b c} F_{b c I J}(A) \approx 0$ are reducible. Dirac's canonical analysis for BF theory with a cosmological constant is reported in the Appendix A, where it is shown that now the reducibility equations involve both the Gauss constraints $\widetilde{\Psi}^{I J}$ and the other set of first class constraints $\widetilde{\Psi}_{I J}^{a}:=\frac{1}{2} \widetilde{\eta}^{a b c} F_{b c I J}-\Lambda \varepsilon_{I J K L} \widetilde{\Pi}^{a K L} \approx 0$. This is so because of the cosmological constant $\Lambda$. In both cases, the reducibility equations in Dirac's canonical analysis come from the Bianchi identities $D F_{I J}=0$. So, the covariant canonical formalism is an opportunity to understand the role these identities play on this formalism.

\section{BF THEORY}

The four-dimensional BF theory with $S O(3,1)$ as the internal relevant group is defined by the equations of motion

$$
F_{I J}=0, \quad D B^{I J}=0
$$

where $F_{I J}(A)=d A_{I J}+A_{I K} \wedge A^{K}{ }_{J}$ is the curvature of the Lorentz connection 1-form $A_{I J}$, $B^{I J}=\frac{1}{2} B_{\alpha \beta}^{I J} d x^{\alpha} \wedge d x^{\beta}$ is a set of six 2-forms, $D B^{I J}:=d B^{I J}+A^{I}{ }_{K} \wedge B^{K J}+A^{J}{ }_{K} \wedge B^{I K}$ is the covariant derivative of $B^{I J} ; I, J=0,1,2,3$ are Lorentz indexes which are raised and lowered 
with the Minkowski metric $\eta_{I J}$. Even though the analysis will be restricted to a Lorentz $\mathrm{BF}$ theory, the results are generic in the sense that hold for any BF theory in 4-spacetime dimensions. The choice of the Lorentz group is only to fix the notation that might be used for the case of BF gravity.

In the context of the covariant canonical formalism, the kinematical phase space $\mathscr{F}$ of the theory is defined as the space formed by all smooth Lorentz connections $A_{I J}$ and $B^{I J}$ fields. Any generic point of $\mathscr{F}$ is not required to satisfy the equations of motion of the BF theory. The space of solutions to the equations of motion $\overline{\mathscr{F}}$ is considered as submanifold of $\mathscr{F}$ and is formed by all points of $\mathscr{F}$ that satisfy the equations of motion of Eq. (1D). The reduced (or physical) phase space for the theory is reached by making the quotient of $\overline{\mathscr{F}}$ by the gauge transformations of the theory $[6,7]$. Even though the term "phase space" has been used to name these different manifolds, it must be emphasized that, at this stage, $\mathscr{F}$, $\overline{\mathscr{F}}$, and the reduced phase space carry no intrinsic symplectic geometry. Thus, although the equations of motion of Eq. (11) are used to define $\overline{\mathscr{F}}$, they are not enough to uniquely endow the various phase spaces for the theory already mentioned with symplectic geometry. Where does symplectic geometry come from then? One possibility is from action principles, specifying the Lagrangian density [13]. The equations of motion of Eq. (11) are usually obtained from the action [1]

$$
S[A, B]=\int_{\mathcal{M}} B^{I J} \wedge F_{I J}[A] .
$$

[see also Refs. 14] and 15] for alternative choices of the action]. To get the geometry, one needs to proceed along the following lines. The first order variation of the Lagrangian 4 -form $\mathbf{L}[A, B]=B^{I J} \wedge F_{I J}[A]$ is

$$
\delta \mathbf{L}[A, B]=\left(\delta B^{I J}\right) \wedge F_{I J}-\left(D B^{I J}\right) \wedge \delta A_{I J}+d \mathbf{\Theta}(B, \delta A),
$$

from which the presymplectic potential 3-form

$$
\Theta(B, \delta A):=B^{I J} \wedge \delta A_{I J},
$$

is read off. Now, by taking into account an arbitrary smooth two-parameter family of field configurations and computing the antisymmetric combination of the variations in $\mathbf{L}[A, B]$, $\left[\delta_{1}, \delta_{2}\right] \mathbf{L}[A, B]=0$ yields []

$$
d \omega\left(\delta_{1} A, \delta_{1} B, \delta_{2} A, \delta_{2} B\right)=\left(\delta_{1} B^{I J}\right) \wedge \delta_{2} F_{I J}-\left(\delta_{2} D B^{I J}\right) \wedge \delta_{1} A_{I J}-\left(\delta_{1} \longleftrightarrow \delta_{2}\right),
$$


where

$$
\omega\left(\delta_{1} A, \delta_{1} B, \delta_{2} A, \delta_{2} B\right)=\left(\delta_{1} B^{I J} \wedge \delta_{2} A_{I J}-\delta_{2} B^{I J} \wedge \delta_{1} A_{I J}\right)
$$

is the presymplectic 3 -form [16].

\section{A. "Fundamental" set of local gauge transformations}

(i) Local Lorentz transformations: The action is fully gauge invariant under any arbitrary finite local Lorentz transformation. The infinitesimal version of this transformation is

$$
\begin{aligned}
& \delta_{\varepsilon} A_{I J}=D \varepsilon_{I J}, \\
& \delta_{\varepsilon} B^{I J}=-\varepsilon^{I}{ }_{K} B^{K J}-\varepsilon^{J}{ }_{K} B^{I K},
\end{aligned}
$$

where $\varepsilon_{I J}$ are the infinitesimal gauge parameters. The change of the Lagrangian $\mathbf{L}[A, B]$ induced by the infinitesimal variation of the fields, given in Eq. (7), is

$$
\begin{aligned}
\delta_{\varepsilon} \mathbf{L}[A, B] & =\delta_{\varepsilon} B^{I J} \wedge F_{I J}+B^{I J} \wedge \delta_{\varepsilon} F_{I J} \\
& =0
\end{aligned}
$$

Therefore, from Eqs. (3) and (8) the Noether current 3 -form $\mathbf{J}_{N}[A, B, \varepsilon]$ (Ref. [8]) associated with the symmetry (17) is [17]

$$
\begin{aligned}
\mathbf{J}_{N}[A, B, \varepsilon] & =\boldsymbol{\Theta}\left(B, D \varepsilon_{I J}\right) \\
& =B^{I J} \wedge D \varepsilon_{I J},
\end{aligned}
$$

which can be rewritten as

$$
\mathbf{J}_{N}[A, B, \varepsilon]=d \mathbf{Q}[B, \varepsilon]-\varepsilon_{I J} \wedge D B^{I J}
$$

with

$$
\mathbf{Q}[B, \varepsilon]:=\varepsilon_{I J} B^{I J},
$$

the corresponding Noether current potential 2-form. Equation (10) has the same structure that appears in the Noether current associated with infinitesimal diffeomorphisms in theories with dynamical background metric in the sense that the right-hand side of Eq. (10) is the 
exterior derivative of the Noether current potential 2-form $\mathbf{Q}[B, \varepsilon]$ plus a term proportional to (one set of) the equations of motion [18]. There is a priori no reason for an internal symmetry, like (7), behaves in the same manner as diffeomorphisms.

Degenerate directions: These can be obtained from the symplectic inner product between the gauge transformation $\delta_{\varepsilon}$ and an arbitrary variation $\delta$ by taking $\delta_{1} \equiv \delta$ and $\delta_{2}=\delta_{\varepsilon}$. From Eqs. (6) and (7),

$$
\begin{aligned}
\omega\left(\delta A, \delta B, \delta_{\varepsilon} A, \delta_{\varepsilon} B\right) & =d\left(\varepsilon_{I J} \delta B^{I J}\right)-\varepsilon_{I J} \delta\left(D B^{I J}\right) \\
& =d(\delta \mathbf{Q}[B, \varepsilon])-\varepsilon_{I J} \delta\left(D B^{I J}\right) .
\end{aligned}
$$

Note that on the right-hand side of Eq. (12) appears one term involving the linearized Eulerian derivative, $\delta\left(D B^{I J}\right)$, but no terms proportional to Eulerian derivatives themselves appear explicitly. As it will be seen, later on, this is a difference with respect to infinitesimal diffeomorphisms (see Sec. IIB). Thus, we have

$$
\begin{aligned}
\Omega_{\Sigma}\left(\delta A, \delta B, \delta_{\varepsilon} A, \delta_{\varepsilon} B\right) & :=\int_{\Sigma} \omega\left(\delta A, \delta B, \delta_{\varepsilon} A, \delta_{\varepsilon} B\right) \\
& =-\int_{\Sigma} \varepsilon_{I J} \delta D B^{I J}+\int_{\partial \Sigma}\left(\varepsilon_{I J} \delta B^{I J}\right) .
\end{aligned}
$$

The integral over $\Sigma$ depends on the gauge parameters $\varepsilon_{I J}$, the fields $\left(A_{I J}, B^{K L}\right)$ and their variations while the integral over $\partial \Sigma$ depends only on the gauge parameters $\varepsilon_{I J}$ and the variation of the $B^{I J}$ fields, $\delta B^{I J}$. Both integrals, in general, do not vanish and therefore the gauge transformation of Eq. (7) does not qualify as a degenerate direction unless additional assumptions are imposed. In particular, one has the following.

Proposition: If the linearized Eulerian derivative $\delta\left(D B^{I J}\right)$ vanishes, $\delta\left(D B^{I J}\right)=0$, and the arbitrary variations $\delta B^{I J}$ have compact support in the interior of $\Sigma,\left.\delta B^{I J}\right|_{\partial \Sigma}=0$, then

$$
\Omega_{\Sigma}\left(\delta A, \delta B, \delta_{\varepsilon} A, \delta_{\varepsilon} B\right)=0
$$

without imposing any additional restrictions on the gauge parameters $\varepsilon_{I J}$. Note that $\left(A_{I J}, B^{K L}\right)$ need not be a point of the space of solutions to the equations of motion $\overline{\mathscr{F}}$ in order for Eq. (14) to hold (see also Ref. [19]). Nevertheless, it is a common fact to restrict the analysis to $\overline{\mathscr{F}}$ and also to take $\left(\delta A_{I J}, \delta B^{K L}\right)$ as tangent vectors to $\overline{\mathscr{F}}$. Of course, the integral over $\partial \Sigma$ in Eq. (13) also vanishes if the gauge parameters $\varepsilon_{I J}$ vanish at $\partial \Sigma$, i.e., if the infinitesimal gauge transformation of Eq. (7) is the identity at $\partial \Sigma$.

Canonical transformations: 
Proposition: The infinitesimal gauge transformation of Eq. (17) is a canonical transformation.

Proof: From the gauge transformation of Eq. (71),

$$
\begin{aligned}
& A_{I J}^{\prime}=A_{I J}+D \varepsilon_{I J}, \\
& B^{\prime I J}=B^{I J}-\varepsilon^{I}{ }_{K} B^{K J}-\varepsilon^{J}{ }_{K} B^{I K},
\end{aligned}
$$

we can compute two arbitrary variations of the gauge-transformed fields of Eq. (15) (Ref. 7]),

$$
\begin{aligned}
& \delta_{i} A^{\prime}{ }_{I J}=\delta_{i} A_{I J}-\delta_{i} A^{K}{ }_{I} \varepsilon_{K J}-\delta_{i} A^{K}{ }_{J} \varepsilon_{I K}, \\
& \delta_{i} B^{\prime J}=\delta_{i} B^{I J}-\varepsilon^{I}{ }_{K} \delta_{i} B^{K J}-\varepsilon^{J}{ }_{K} \delta_{i} B^{I K} .
\end{aligned}
$$

So,

$$
\begin{aligned}
\omega^{\prime}:= & \left(\delta_{1} B^{\prime I J} \wedge \delta_{2} A^{\prime}{ }_{I J}-\delta_{2} B^{\prime I J} \wedge \delta_{1} A^{\prime}{ }_{I J}\right) \\
= & \left(\delta_{1} B^{I J}-\varepsilon^{I}{ }_{K} \delta_{1} B^{K J}-\varepsilon^{J}{ }_{K} \delta_{1} B^{I K}\right) \wedge\left(\delta_{2} A_{I J}-\delta_{2} A^{K}{ }_{I} \varepsilon_{K J}-\delta_{2} A^{K}{ }_{J} \varepsilon_{I K}\right) \\
& -\left(\delta_{2} B^{I J}-\varepsilon^{I}{ }_{K} \delta_{2} B^{K J}-\varepsilon^{J}{ }_{K} \delta_{2} B^{I K}\right) \wedge\left(\delta_{1} A_{I J}-\delta_{1} A^{K}{ }_{I} \varepsilon_{K J}-\delta_{1} A^{K}{ }_{J} \varepsilon_{I K}\right) \\
= & \omega,
\end{aligned}
$$

exactly, i.e., without using any additional conditions. Therefore,

$$
\begin{aligned}
\Omega_{\Sigma}^{\prime} & :=\int_{\Sigma} \omega\left(\delta_{1} A^{\prime}, \delta_{1} B^{\prime}, \delta_{2} A^{\prime}, \delta_{2} B^{\prime}\right) \\
& =\int_{\Sigma} \omega\left(\delta_{1} A, \delta_{1} B, \delta_{2} A, \delta_{2} B\right)=\Omega_{\Sigma}
\end{aligned}
$$

(ii) B's transform like connections: The infinitesimal version of this gauge transformation is

$$
\begin{aligned}
& \delta_{\chi} A_{I J}=0 \\
& \delta_{\chi} B^{I J}=D \chi^{I J},
\end{aligned}
$$

where the gauge parameters $\chi^{I J}$ are 1 -forms. However, this symmetry is peculiar in the sense that it does not satisfy the definition of symmetry in a strict sense [8]. To see this, the variation of the Lagrangian $\mathbf{L}[A, B]$ induced by the variation of the fields is computed

$$
\delta_{\chi} \mathbf{L}[A, B]=D \chi^{I J} \wedge F_{I J}
$$


which has not the desired form in the sense that the right-hand side of Eq. (20) is not of the form $d \boldsymbol{\alpha}$. To continue, we must rewrite the right-hand side of the last equation,

$$
\delta_{\chi} \mathbf{L}[A, B]=d\left(\chi^{I J} \wedge F_{I J}\right)+\chi^{I J} \wedge D F_{I J}
$$

Thus, the right-hand side of Eq. (21) is not, in a strict sense, of the form $d \boldsymbol{\alpha}$. It acquires this form just if the Bianchi identities $D F_{I J}=0$ are used. However, when computing the transformation of the Lagrangian $\mathbf{L}[A, B]$ induced by the transformation of the fields it is not allowed to use the equations of motion in order to check if the transformation of the fields does (or does not) qualify as a gauge symmetry. A purist might say that the second term on the right-hand side of Eq. (21) involves no equations of motion simply because the Bianchi identities do not qualify as equations of motion in the sense that they do not appear when the first order variation of the Lagrangian is computed [see Eq. (3)] ].

Therefore, from Eqs. (3) and (21),

$$
d\left(\chi^{I J} \wedge F_{I J}\right)+\chi^{I J} \wedge D F_{I J}=\left(D \chi^{I J}\right) \wedge F_{I J}
$$

and so

$$
d \mathbf{J}_{N}[A, \chi]=-\chi^{I J} \wedge D F_{I J}+\left(D \chi^{I J}\right) \wedge F_{I J}
$$

with [17]

$$
\mathbf{J}_{N}[A, \chi]:=\chi^{I J} \wedge F_{I J}
$$

the Noether current associated with the local symmetry (19). Note that $\mathbf{J}_{N}[A, \chi]$ is proportional to the Eulerian derivative $F_{I J}$. Note that if the equations of motion hold (i.e., if $F_{I J}=0$ hold) and the Bianchi identities hold (i.e, if $D F_{I J}=0$ hold) then the Noether current is identically conserved. Moreover, note that $\mathbf{J}_{N}[A, \chi]$ identically vanishes on-shell, i.e., $\mathbf{J}_{N}=0$ if $F_{I J}=0$.

Degenerate directions: Again, from the symplectic inner product between the gauge transformation $\delta_{\chi}$ and an arbitrary variation $\delta$ and Eqs. (6) and (19)

$$
\omega\left(\delta A, \delta B, \delta_{\chi} A, \delta_{\chi} B\right)=d\left(\delta\left(-\chi^{I J} \wedge A_{I J}\right)\right)-\chi^{I J} \wedge \delta F_{I J}
$$

Note that on the right-hand side of Eq. (25) appears the linearized Eulerian derivative $\delta F_{I J}$ but not the Eulerian derivatives themselves in contrast to what happens with diffeomor- 
phisms (see Sec. 【B). Thus, we have

$$
\begin{aligned}
\Omega_{\Sigma}\left(\delta A, \delta B, \delta_{\chi} A, \delta_{\chi} B\right) & :=\int_{\Sigma} \omega\left(\delta A, \delta B, \delta_{\chi} A, \delta_{\chi} B\right) \\
& =-\int_{\Sigma} \chi^{I J} \wedge \delta F_{I J}-\int_{\partial \Sigma}\left(\chi^{I J} \wedge \delta A_{I J}\right) .
\end{aligned}
$$

Again, the integral over $\Sigma$ depends on the gauge parameters $\chi^{I J}$, the field $A_{I J}$ and its first order variations $\delta A_{I J}$ while the integral over $\partial \Sigma$ depends only on the gauge parameters $\chi^{I J}$ and the variations of the field $A_{I J}, \delta A_{I J}$. Both integrals, in general, do not vanish and therefore the gauge transformation of Eq. (19) does not qualify as a degenerate direction unless additional assumptions are imposed. In particular, one has the following.

Proposition. If the linearized Eulerian derivative $\delta F_{I J}$ vanishes, $\delta F_{I J}=0$, and the arbitrary variations $\delta A_{I J}$ have compact support in the interior of $\Sigma,\left.\delta A_{I J}\right|_{\partial \Sigma}=0$, then

$$
\Omega_{\Sigma}\left(\delta A, \delta B, \delta_{\chi} A, \delta_{\chi} B\right)=0,
$$

without imposing any additional conditions on the gauge parameters $\chi^{I J}$. Note also that in order for Eq. (27) to hold it is not necessary that the point $\left(A_{I J}, B^{K L}\right)$ belongs to the space of solutions to the equations of motion $\overline{\mathscr{F}}$. Nevertheless, it is a common fact to restrict the analysis to this case and also to take $\left(\delta A_{I J}, \delta B^{K L}\right)$ as tangent vectors to $\overline{\mathscr{F}}$. Of course, the integral over $\partial \Sigma$ in Eq. (26) also vanishes if the gauge parameters $\chi^{I J}$ vanish at $\partial \Sigma$, i.e., if the infinitesimal gauge transformation of Eq. (19) is the identity at $\partial \Sigma$.

Canonical transformations:

Proposition: The transformation induced by the gauge symmetry of Eq. (19) is an infinitesimal canonical transformation.

Proof: In fact, from Eq. (19),

$$
\begin{aligned}
& A_{I J}^{\prime}=A_{I J}, \\
& B^{\prime I J}=B^{I J}+D \chi^{I J},
\end{aligned}
$$

we can compute two arbitrary variations of the gauge-transformed fields [7]

$$
\begin{aligned}
& \delta_{i} A_{I J}^{\prime}=\delta_{i} A_{I J}, \\
& \delta_{i} B^{\prime I J}=\delta_{i} B^{I J}+\delta_{i} A_{K}^{I} \wedge \chi^{K J}+\delta_{i} A^{J}{ }_{K} \wedge \chi^{I K}, \quad i=1,2 .
\end{aligned}
$$

So,

$$
\omega^{\prime}:=\left(\delta_{1} B^{\prime I J} \wedge \delta_{2} A_{I J}^{\prime}-\delta_{2} B^{\prime I J} \wedge \delta_{1} A_{I J}^{\prime}\right)
$$




$$
\begin{aligned}
= & \left(\delta_{1} B^{I J}+\delta_{1} A_{K}^{I} \wedge \chi^{K J}+\delta_{1} A^{J}{ }_{K} \wedge \chi^{I K}\right) \wedge \delta_{2} A_{I J} \\
& -\left(\delta_{2} B^{I J}+\delta_{2} A_{K}^{I} \wedge \chi^{K J}+\delta_{2} A^{J}{ }_{K} \wedge \chi^{I K}\right) \wedge \delta_{1} A_{I J} \\
= & \omega
\end{aligned}
$$

exactly, i.e., without using any additional conditions. Therefore,

$$
\begin{aligned}
\Omega_{\Sigma}^{\prime} & :=\int_{\Sigma} \omega\left(\delta_{1} A^{\prime}, \delta_{1} B^{\prime}, \delta_{2} A^{\prime}, \delta_{2} B^{\prime}\right) \\
& =\int_{\Sigma} \omega\left(\delta_{1} A, \delta_{1} B, \delta_{2} A, \delta_{2} B\right)=\Omega_{\Sigma},
\end{aligned}
$$

under the infinitesimal gauge transformation of Eq. (19).

\section{B. Diffeomorphisms}

The gauge symmetries discussed in Sec. ЏA can also be obtained by using Dirac's canonical analysis. In addition, Dirac's canonical analysis shows that the full set of constraints are first class. There are no second class constraints in the theory. However, the first class constraints $\widetilde{\Psi}_{I J}^{a}$ (which generate the $\delta_{\chi}$ symmetry) are reducible on account of the Bianchi identities $D F_{I J}=0$ which imply the reducibility equation $D_{a} \widetilde{\Psi}^{a}{ }_{I J}=0$. Once reducibility is taken into account the counting of the local degrees of freedom is zero, showing that the theory has only global degrees of freedom (see, for instance, Ref. 12] and the Appendix A). Moreover, it is also known that the theory is diffeomorphism covariant. Therefore, the transformation of the fields induced by diffeomorphisms must be built from the "fundamental" set of gauge transformations (77) and (19). (The quotation marks in the word "fundamental" emphasize the fact that the gauge transformations are not independent on account of the reducibility of the constraints.) In fact, a diffeomorphism induces a change in the fields $A_{I J}$ given by

$$
\delta_{\xi} A_{I J}=\mathcal{L}_{\xi} A_{I J}=\xi \cdot F_{I J}+D \varepsilon_{I J}=\xi \cdot F_{I J}+\delta_{\varepsilon} A_{I J},
$$

as well as in the fields $B^{I J}$,

$$
\begin{aligned}
\delta_{\xi} B^{I J} & =\mathcal{L}_{\xi} B^{I J}=\xi \cdot D B^{I J}-\varepsilon^{I}{ }_{K} B^{K J}-\varepsilon^{J}{ }_{K} B^{I K}+D \chi^{I J} \\
& =\xi \cdot D B^{I J}+\delta_{\varepsilon} B^{I J}+\delta_{\chi} B^{I J},
\end{aligned}
$$

where $\varepsilon_{I J}:=\xi \cdot A_{I J}$ is a set of 0 -forms and $\chi^{I J}:=\xi \cdot B^{I J}$ is a set of 1 -forms. 
Noether current: The Noether current 3-form associated with diffeomorphisms is [17]

$$
\begin{aligned}
\mathbf{J}_{N}[A, B, \xi] & =\boldsymbol{\Theta}\left(B, \mathcal{L}_{\xi} A\right)-\xi \cdot \mathbf{L} \\
& =d \mathbf{Q}[A, B, \xi]-\left(\xi \cdot A_{I J}\right) D B^{I J}-\left(\xi \cdot B^{I J}\right) \wedge F_{I J}
\end{aligned}
$$

where

$$
\mathbf{Q}[A, B, \xi]=\left(\xi \cdot A_{I J}\right) B^{I J}
$$

is the Noether current potential 2-form. If $\left(A_{I J}, B^{I J}\right)$ is a point of the space of solutions to the equations of motion $\overline{\mathscr{F}}$ then $\mathbf{J}_{N}[A, B, \xi]$ can be obtained from the Noether current potential $\mathbf{J}_{N}[A, B, \xi]=d \mathbf{Q}[A, B, \xi]$. The Noether charge $\mathcal{Q}_{\Sigma}(\xi)$ associated with infinitesimal diffeomorphisms is given by the integral of $\mathbf{J}_{N}[A, B, \xi]$ over $\Sigma$,

$$
\begin{aligned}
\mathcal{Q}_{\Sigma}(\xi) & :=\int_{\Sigma} \mathbf{J}_{N}[A, B, \xi] \\
& =\int_{\partial \Sigma} \mathbf{Q}[A, B, \xi]-\int_{\Sigma}\left[\left(\xi \cdot A_{I J}\right) D B^{I J}+\left(\xi \cdot B^{I J}\right) \wedge F_{I J}\right] .
\end{aligned}
$$

Proposition: If $\left(A_{I J}, B^{I J}\right)$ is a point in $\overline{\mathscr{F}}$ then the Noether charge just has a contribution from the boundary of $\Sigma$,

$$
\mathcal{Q}_{\Sigma}(\xi)=\int_{\partial \Sigma} \mathbf{Q}[A, B, \xi]=\int_{\partial \Sigma}\left(\xi \cdot A_{I J}\right) B^{I J}
$$

Canonical transformation induced by diffeomorphisms: The transformation of the fields induced by a diffeomorphism is

$$
\begin{aligned}
& A^{\prime}{ }_{I J}=A_{I J}+\mathcal{L}_{\xi} A_{I J}, \\
& B^{\prime I}=B^{I J}+\mathcal{L}_{\xi} B^{I J},
\end{aligned}
$$

and so [7]

$$
\begin{aligned}
& \delta_{i} A^{\prime}{ }_{I J}=\delta_{i} A_{I J}+\delta_{i}\left(\mathcal{L}_{\xi} A_{I J}\right)=\delta_{i} A_{I J}+\mathcal{L}_{\xi}\left(\delta_{i} A_{I J}\right) \\
& \delta_{i} B^{\prime I}=\delta_{i} B^{I J}+\delta_{i}\left(\mathcal{L}_{\xi} B^{I J}\right)=\delta_{i} B^{I J}+\mathcal{L}_{\xi}\left(\delta_{i} B^{I J}\right)
\end{aligned}
$$

Therefore, at first order in the gauge parameters,

$$
\begin{aligned}
\omega^{\prime} & :=\left(\delta_{1} B^{\prime I J} \wedge \delta_{2} A^{\prime}{ }_{I J}-\delta_{2}{B^{\prime}}^{I J} \wedge \delta_{1} A^{\prime}{ }_{I J}\right) \\
& =\omega+\mathcal{L}_{\xi} \omega .
\end{aligned}
$$


Finally,

$$
\Omega_{\Sigma}^{\prime}:=\int_{\Sigma} \omega^{\prime}=\int_{\Sigma} \omega+\int_{\Sigma}(\xi \cdot d \omega+d(\xi \cdot \omega))=\Omega_{\Sigma}+\int_{\Sigma}(\xi \cdot d \omega+d(\xi \cdot \omega))
$$

If the linearized Eulerian derivatives vanish, i.e., if $\delta_{i}\left(F_{I J}\right)=0$ and $\delta_{i}\left(D B^{I J}\right)=0, i=1,2$ then $d \omega=0$, and so

$$
\Omega_{\Sigma}^{\prime}=\Omega_{\Sigma}+\int_{\Sigma} d(\xi \cdot \omega)=\Omega_{\Sigma}+\int_{\partial \Sigma} \xi \cdot \omega
$$

Note that $A_{I J}$ and $B^{I J}$ in $\delta_{i}\left(F_{I J}\right)=0$ and $\delta_{i}\left(D B^{I J}\right)=0$ are $n o t$ required to be solutions to the equations of motion $F_{I J}=0$ and $D B^{I J}=0$, i.e., $\delta_{i} A_{I J}$ and $\delta_{i} B^{I J}$ are tangent to $\mathscr{F}$ but not to $\overline{\mathscr{F}}$. Thus, if

$$
\int_{\partial \Sigma} \xi \cdot \omega=0
$$

then

$$
\Omega_{\Sigma}^{\prime}=\Omega_{\Sigma}
$$

This result can be summarized in the following:

Proposition: If the linearized Eulerian derivatives $\delta_{i}\left(F_{I J}\right)=0$ and $\delta_{i}\left(D B^{I J}\right)=0$ hold then Eq. (43) is a necessary and sufficient condition for $\Omega_{\Sigma}$ be invariant under the transformation associated with infinitesimal diffeomorphisms, i.e., if the linearized Eulerian derivatives $\delta_{i}\left(F_{I J}\right)=0$ and $\delta_{i}\left(D B^{I J}\right)=0$ hold then Eq. (43) is a necessary and sufficient condition for infinitesimal diffeomorphisms to be canonical transformations.

It is clear that in the particular case when $\Sigma$ has no boundary, i.e., $\partial \Sigma=\emptyset$ then Eq. (43) holds without any additional restrictions on $\xi$.

Degenerate directions: The starting point is the expression for the presymplectic 3-form with $\delta_{1}=\delta$ an arbitrary variation and $\delta_{2}$ is taken as the variation induced by the Lie derivative on the dynamical fields. From Eqs. (66) and (38),

$$
\omega\left(\delta A, \delta B, \mathcal{L}_{\xi} A, \mathcal{L}_{\xi} B\right)=\left(\delta B^{I J} \wedge \mathcal{L}_{\xi} A_{I J}-\mathcal{L}_{\xi}\left(B^{I J} \wedge \delta A_{I J}\right)+B^{I J} \wedge \delta\left(\mathcal{L}_{\xi} A_{I J}\right)\right)
$$

Now, by taking $\delta=\mathcal{L}_{\xi}$ in the expression for the presymplectic current potential 3-form of Eq. (41) and computing its variation one has

$$
\delta \Theta\left(B, \mathcal{L}_{\xi} A\right)=\delta B^{I J} \wedge \mathcal{L}_{\xi} A_{I J}+B^{I J} \wedge \delta\left(\mathcal{L}_{\xi} A_{I J}\right)
$$


Inserting the right-hand side of Eq. (46) into the right-hand side of Eq. (45) one gets

$$
\begin{aligned}
\omega\left(\delta A, \delta B, \mathcal{L}_{\xi} A, \mathcal{L}_{\xi} B\right) & =\delta \Theta\left(B, \mathcal{L}_{\xi} A\right)-\mathcal{L}_{\xi}\left(B^{I J} \wedge \delta A_{I J}\right) \\
& =\delta \Theta\left(B, \mathcal{L}_{\xi} A\right)-\mathcal{L}_{\xi} \Theta(B, \delta A)
\end{aligned}
$$

On the other hand, the variation of the Noether current 3 -form is

$$
\begin{aligned}
\delta \mathbf{J}_{N}[A, B, \xi]= & \delta \boldsymbol{\Theta}\left(B, \mathcal{L}_{\xi} A\right)-\xi \cdot \delta \mathbf{L} \\
= & \delta \boldsymbol{\Theta}\left(B, \mathcal{L}_{\xi} A\right)-\xi \cdot\left(\left(\delta B^{I J}\right) \wedge F_{I J}-\left(D B^{I J}\right) \wedge \delta A_{I J}+d \boldsymbol{\Theta}(B, \delta A)\right) \\
= & \omega\left(\delta A, \delta B, \mathcal{L}_{\xi} A, \mathcal{L}_{\xi} B\right)+\mathcal{L}_{\xi} \boldsymbol{\Theta}(B, \delta A) \\
& -\xi \cdot\left(\left(\delta B^{I J}\right) \wedge F_{I J}-\left(D B^{I J}\right) \wedge \delta A_{I J}\right)-\xi \cdot d \boldsymbol{\Theta}(B, \delta A) \\
= & \omega\left(\delta A, \delta B, \mathcal{L}_{\xi} A, \mathcal{L}_{\xi} B\right)-\xi \cdot\left(\left(\delta B^{I J}\right) \wedge F_{I J}-\left(D B^{I J}\right) \wedge \delta A_{I J}\right) \\
& +d(\xi \cdot \boldsymbol{\Theta}(B, \delta A)) .
\end{aligned}
$$

To get the second line on the right-hand side, Eq. (3) was used while Eq. (47) was used to get the third line. Inserting the explicit expression for $\delta \mathbf{J}_{N}[A, B, \xi]$ given in Eq. (34) into the left-hand side of Eq. (48) one has

$$
\begin{aligned}
\omega\left(\delta A, \delta B, \mathcal{L}_{\xi} A, \mathcal{L}_{\xi} B\right)= & d\left(\delta \mathbf{Q}_{N}[A, B, \xi]-\xi \cdot \boldsymbol{\Theta}(B, \delta A)\right)-\left(\xi \cdot A_{I J}\right) \delta D B^{I J} \\
& -\left(\xi \cdot B^{I J}\right) \wedge \delta F_{I J}+\left(\delta B^{I J}\right) \wedge\left(\xi \cdot F_{I J}\right) \\
& -\left(\xi \cdot D B^{I J}\right) \wedge \delta A_{I J}
\end{aligned}
$$

Note that, in contrast to Eqs. (12) and (25), in the case of diffeomorphisms the symplectic inner product between $\delta_{\xi}$ and an arbitrary variation $\delta$ involves both Eulerian derivatives and the linearized Eulerian derivatives. One has the following:

Proposition: Let $\left(A_{I J}, B^{I J}\right)$ be a point in $\overline{\mathscr{F}}$; let $\left(\delta A_{I J}, \delta B^{I J}\right)$ be a solution to the linearized Eulerian derivatives at $\left(A_{I J}, B^{I J}\right)$ [i.e., $\left(\delta A_{I J}, \delta B^{I J}\right)$ are such that $\delta\left(D B^{I J}\right)=0$ and $\delta F_{I J}=0$ and are tangent to $\overline{\mathscr{F}}$ at $\left.\left(A_{I J}, B^{I J}\right)\right]$. Then, we have

$$
\omega\left(\delta A, \delta B, \mathcal{L}_{\xi} A, \mathcal{L}_{\xi} B\right)=d\left(\delta \mathbf{Q}_{N}[A, B, \xi]-\xi \cdot \boldsymbol{\Theta}(B, \delta A)\right)
$$

and thus, integrating on $\Sigma$,

$$
\begin{aligned}
\Omega_{\Sigma}\left(\delta A, \delta B, \mathcal{L}_{\xi} A, \mathcal{L}_{\xi} B\right): & =\int_{\Sigma} \omega\left(\delta A, \delta B, \mathcal{L}_{\xi} A, \mathcal{L}_{\xi} B\right) \\
& =\oint_{\partial \Sigma}\left(\delta \mathbf{Q}_{N}[A, B, \xi]-\xi \cdot \boldsymbol{\Theta}(B, \delta A)\right)
\end{aligned}
$$


Inserting the explicit expressions for $\mathbf{Q}_{N}[A, B, \xi]$ and $\boldsymbol{\Theta}(B, \delta A)$ one has

$$
\begin{aligned}
\Omega_{\Sigma}\left(\delta A, \delta B, \mathcal{L}_{\xi} A, \mathcal{L}_{\xi} B\right) & =\oint_{\partial \Sigma}\left[\delta\left(\left(\xi \cdot A_{I J}\right) B^{I J}\right)-\xi \cdot\left(B^{I J} \wedge \delta A_{I J}\right)\right] \\
& =\oint_{\partial \Sigma}\left[\left(\xi \cdot A_{I J}\right) \delta B^{I J}-\left(\xi \cdot B^{I J}\right) \wedge \delta A_{I J}\right] .
\end{aligned}
$$

Some remarks follow: (1) first of all, Eq. (52) tells us that, in the context of the covariant canonical formalism, not all diffeomorphisms are to be regarded as gauge because the righthand side of (52) will not vanish for any $\xi$, (2) note that if $\left(A_{I J}, B^{I J}\right) \in \overline{\mathscr{F}}$ then $\xi \cdot A_{I J} \neq 0$ and $\xi \cdot B^{I J} \neq 0$ in the generic case. Moreover, note that the right-hand side of (152) vanishes for all $\left(A_{I J}, B^{I J}\right)$ of $\overline{\mathscr{F}}$ and for all tangent variation $\left(\delta A_{I J}, \delta B^{I J}\right)$ to $\overline{\mathscr{F}}$ in $\left(A_{I J}, B^{I J}\right)$ if and only if $\xi$ vanishes at the boundary $\partial \Sigma,\left.\xi\right|_{\partial \Sigma}=0$. Thus, just those diffeomorphisms which are the identity at $\partial \Sigma$ must be regarded as gauge. One could say that the gauge transformation is broken at $\partial \Sigma$ in the sense that $\left.\xi\right|_{\partial \Sigma}=0$. However, from that perspective one would be $a$ priori assuming that all diffeomorphisms are gauge which as the previous analysis shows is not the case. Let $\zeta$ be a diffeomorphism such that it does not vanish at $\partial \Sigma$. The full set of these $\zeta$ 's span the boundary symmetry group. Thus, the covariant canonical formalism tells us that the boundary symmetry group is not a gauge group (see also Ref. [9] to understand the role of diffeomorphisms in the case of general relativity).

Existence of a Hamiltonian: For variations $\delta A_{I J}$ with compact support in the interior of $\Sigma$, i.e., $\left.\delta A_{I J}\right|_{\partial \Sigma}=0$,

$$
\Omega_{\Sigma}=\delta \oint_{\partial \Sigma}\left(\zeta \cdot A_{I J}\right) B^{I J}=\delta \oint_{\partial \Sigma} \mathbf{Q}[A, B, \zeta]
$$

which means that a Hamiltonian conjugate to $\zeta$ on $\Sigma$ exists and that its Hamiltonian density is precisely the Noether potential $\mathbf{Q}[A, B, \zeta]$. We have assumed that $\zeta$ does not vanish at $\partial \Sigma$ and thus, by definition, $\zeta$ is not a degenerate direction.

On the other hand, for variations $\delta B_{I J}$ with compact support in the interior of $\Sigma$, i.e., $\left.\delta B^{I J}\right|_{\partial \Sigma}=0$,

$$
\Omega_{\Sigma}=\delta \oint_{\partial \Sigma}-\left(\zeta \cdot B^{I J}\right) \wedge A_{I J}
$$

so there exists a Hamiltonian conjugate to $\zeta$ on $\Sigma$.

Relationship between the Noether currents: It is possible to compare the Noether current associated with diffeomorphisms, $\mathbf{J}_{N}[A, B, \xi]$, with the currents associated to the fundamen- 
tal set of gauge symmetries

$$
\begin{aligned}
\mathbf{J}_{N}[A, B, \xi] & =B^{I J} \wedge \mathcal{L}_{\xi} A_{I J}-\xi \cdot\left(B^{I J} \wedge F_{I J}\right) \\
& =B^{I J} \wedge\left[\xi \cdot F_{I J}+D \varepsilon_{I J}\right]-\left(\xi \cdot B^{I J}\right) \wedge F_{I J}-B^{I J} \wedge\left(\xi \cdot F_{I J}\right) \\
& =B^{I J} \wedge D \varepsilon_{I J}-\chi^{I J} \wedge F_{I J} \\
& =\mathbf{J}_{N}[A, B, \varepsilon]-\mathbf{J}_{N}[A, \chi] .
\end{aligned}
$$

In a more appropriate notation

$$
\mathbf{J}_{N}[A, B, \xi]=\left.\left(\mathbf{J}_{N}[A, B, \varepsilon]-\mathbf{J}_{N}[A, \chi]\right)\right|_{\varepsilon_{I J}=\xi \cdot A_{I J}, \chi^{I J}=\xi \cdot B^{I J}}
$$

Note also that $\mathbf{J}_{N}[A, B, \xi]=\mathbf{J}_{N}[A, B, \varepsilon]$ because $\mathbf{J}_{N}[A, \chi]=0$ on-shell (i.e., if $F_{I J}=0$ ). Moreover, note that

$$
\mathbf{Q}[A, B, \xi]=\left(\xi \cdot A_{I J}\right) B^{I J}=\varepsilon_{I J} B^{I J}=\mathbf{Q}[B, \varepsilon]
$$

\section{BF THEORY PLUS A COSMOLOGICAL CONSTANT}

The four-dimensional BF theory with $S O(3,1)$ as the internal group and supplemented with a cosmological constant $\Lambda$ is defined by the equations of motion

$$
F_{I J}=2 \Lambda * B_{I J}, \quad D B_{I J}=0
$$

where $* B_{I J}=\frac{1}{2} \epsilon_{I J K L} B^{K L}$ is the dual of $B^{I J}$. If $S O(4)$ were taken as the internal group then $\eta_{I J} \longrightarrow \delta_{I J}$, the connection were valued in the Lie algebra of $S O(4)$ and $*^{2}=+1$. Equations (158) can be obtained from the action principle [11, 20]

$$
S[A, B]=\int_{\mathcal{M}} B^{I J} \wedge F_{I J}[A]-\Lambda \int_{\mathcal{M}} B^{I J} \wedge * B_{I J}
$$

Thus, in contrast to BF theory, the space of solutions to the equations of motion $\overline{\mathscr{F}}_{B F+\Lambda}$ is now defined by Eq. (58). To get the geometry, one needs to compute the first order variation of the Lagrangian 4-form $\mathbf{L}[A, B]=B^{I J} \wedge F_{I J}[A]-\Lambda B^{I J} \wedge * B_{I J}$,

$$
\delta \mathbf{L}[A, B]=\left(\delta B^{I J}\right) \wedge\left(F_{I J}-2 \Lambda * B_{I J}\right)-\left(D B^{I J}\right) \wedge \delta A_{I J}+d \mathbf{\Theta}(B, \delta A),
$$

from which the presymplectic potential 3-form

$$
\Theta(B, \delta A):=B^{I J} \wedge \delta A_{I J},
$$


is read off. Therefore, the presymplectic 3 -form $\omega$ is the same of the BF theory. The symplectic structure induced on $\overline{\mathscr{F}}_{B F+\Lambda}$ is simply the pullback to $\overline{\mathscr{F}}_{B F+\Lambda}$ of the curl of $\Theta$ on the kinematical phase space.

\section{Degenerate directions:}

1. The symplectic inner product between $\delta_{1}=\delta$ and $\delta_{2}=\delta_{\varepsilon}, \omega\left(\delta A, \delta B, \delta_{\varepsilon} A, \delta_{\varepsilon} B\right)$, has the same analytical form of the BF theory.

2. The symplectic inner product between $\delta_{1}=\delta$ and $\delta_{2}=\delta_{\chi}$ where $\delta_{\chi} A_{I J}=\Lambda \varepsilon_{I J K L} \chi^{K L}$ and $\delta_{\chi} B^{I J}=D \chi^{I J}$ (Ref. [17]) is now

$$
\omega\left(\delta A, \delta B, \delta_{\chi} A, \delta_{\chi} B\right)=d\left(\delta\left(-\chi^{I J} \wedge A_{I J}\right)\right)-\chi^{I J} \wedge \delta\left(F_{I J}-2 \Lambda * B_{I J}\right)
$$

3. The Noether current associated with diffeomorphisms acquires the form [17]

$$
\mathbf{J}_{N}[A, B, \xi]=d \mathbf{Q}_{N}[A, B, \xi]-\left(\xi \cdot A_{I J}\right) D B^{I J}-\left(\xi \cdot B^{I J}\right) \wedge\left(F_{I J}-2 \Lambda * B_{I J}\right)
$$

with the Noether current potential 2-form $\mathbf{Q}_{N}[A, B, \xi]$ having the same analytical form than the one of the BF theory. Due to the fact that $\omega\left(\delta A, \delta B, \mathcal{L}_{\xi} A, \mathcal{L}_{\xi} B\right)=$ $d\left(\delta \mathbf{Q}_{N}[A, B, \xi]-\xi \cdot \boldsymbol{\Theta}(B, \delta A)\right)$ if the equations of motion and the linearized Eulerian derivatives hold and because $\mathbf{Q}_{N}[A, B, \xi]$ and $\boldsymbol{\Theta}(B, \delta A)$ have the same form of the BF theory, then analysis of the degenerate directions is the same of the BF theory. Finally, note that now $\delta_{\xi}, \delta_{\varepsilon}$, and $\delta_{\chi}$ are not related by (32) but by $\delta_{\xi} A_{I J}=\xi$. $\left(F_{I J}-2 \Lambda * B_{I J}\right)+\delta_{\varepsilon} A_{I J}+\delta_{\chi} A_{I J}$ while $\delta_{\xi} B^{I J}=\xi \cdot D B^{I J}+\delta_{\varepsilon} B^{I J}+\delta_{\chi} B^{I J}$ retains his form with $\varepsilon_{I J}=\xi \cdot A_{I J}, \chi^{I J}=\xi \cdot B^{I J}$.

\section{BF THEORY PLUS THE SECOND CHERN CHARACTER}

Now, the action we consider is the action for BF theory supplemented with the second Chern character

$$
S[A, B]=\int_{\mathcal{M}} B^{I J} \wedge F_{I J}[A]+\theta \int_{\mathcal{M}} F^{I J}[A] \wedge F_{I J}[A]
$$

with $\theta$ a parameter. The first order variation of the Lagrangian 4-form $\mathbf{L}[A, B]=B^{I J} \wedge$ $F_{I J}+\theta F^{I J} \wedge F_{I J}$ is

$$
\delta \mathbf{L}[A, B]=\delta B^{I J} \wedge F_{I J}-\left(D B^{I J}+2 \theta D F^{I J}\right) \wedge \delta A_{I J}+d \mathbf{\Theta}
$$


where

$$
\Theta=\left(B^{I J}+2 \theta F^{I J}\right) \wedge \delta A_{I J}
$$

is the presymplectic potential 3-form [cf. Eq. (44)]. From Eq. (65), it is clear that the equations of motion $F_{I J}=0$ and $D B^{I J}+2 \theta D F^{I J}=0$ coming from Eq. (64) reduce to those of the BF theory because the Bianchi identities $D F_{I J}=0$ always hold. Thus, the inclusion of the second Chern character does not modify the classical dynamics of the BF theory, as expected. This means that the space of solutions to the equations of motion $\overline{\mathscr{F}}$ is the same for both theories. Nevertheless, in spite of the fact that the equations of motion are the same, the presymplectic 3-form changes. For the present case one has

$$
\omega=\delta_{1}\left(B^{I J}+2 \theta F^{I J}\right) \wedge \delta_{2} A_{I J}-\delta_{2}\left(B^{I J}+2 \theta F^{I J}\right) \wedge \delta_{1} A_{I J}
$$

[cf. Eq. (66)]. Therefore, the presymplectic 3-forms coming from Eqs. (21) and (64) are distinct. On the space of solutions $\overline{\mathscr{F}}$, the symplectic structure of Eq. (67) is the same as the symplectic structure of Eq. (6) , of course (see also Ref. [15]).

Degenerate directions:

1. The symplectic inner product between $\delta_{1}=\delta$ and $\delta_{2}=\delta_{\varepsilon}$ is now

$$
\omega\left(\delta A, \delta B, \delta_{\varepsilon} A, \delta_{\varepsilon} B\right)=d\left(\varepsilon_{I J} \delta B^{I J}+2 \theta \delta A^{I J} \wedge D \varepsilon_{I J}\right)-\varepsilon_{I J} \delta\left(D B^{I J}\right)
$$

and so

$$
\Omega_{\Sigma}\left(\delta A, \delta B, \delta_{\varepsilon} A, \delta_{\varepsilon} B\right)=-\int_{\Sigma} \varepsilon_{I J} \delta D B^{I J}+\int_{\partial \Sigma}\left(\varepsilon_{I J} \delta B^{I J}+2 \theta \delta A^{I J} \wedge D \varepsilon_{I J}\right)
$$

Therefore, if the linearized Eulerian derivative $\delta\left(D B^{I J}\right)$ vanishes, $\delta\left(D B^{I J}\right)=0$, and the arbitrary variations $\left(\delta A^{I J}, \delta B^{I J}\right)$ have compact support in the interior of $\Sigma,\left.\delta A^{I J}\right|_{\partial \Sigma}=0$ and $\left.\delta B^{I J}\right|_{\partial \Sigma}=0$, then

$$
\Omega_{\Sigma}\left(\delta A, \delta B, \delta_{\varepsilon} A, \delta_{\varepsilon} B\right)=0
$$

without imposing any additional restrictions on the gauge parameters $\varepsilon_{I J}$. The integral over $\partial \Sigma$ in Eq. (69) also vanishes if both the gauge parameters $\varepsilon_{I J}$ vanish and satisfy $D \varepsilon_{I J}=0$ at $\partial \Sigma$.

2. The symplectic inner product between $\delta_{1}=\delta$ and $\delta_{2}=\delta_{\chi}$ is the same as the one of the BF theory. 
3. Now, we consider diffeomorphisms. The Noether current associated with diffeomorphisms acquires the form

$$
\mathbf{J}_{N}[A, B, \xi]=d \mathbf{Q}_{N}[A, B, \xi]-\left(\xi \cdot A_{I J}\right)\left(D B^{I J}+2 \theta D F^{I J}\right)-\left(\xi \cdot B^{I J}\right) \wedge F_{I J}
$$

where

$$
\mathbf{Q}_{N}[A, B, \xi]=\left(\xi \cdot A_{I J}\right)\left(B^{I J}+2 \theta F^{I J}\right),
$$

is the Noether current potential 2-form. Therefore, the Noether charge is the same as in the $\mathrm{BF}$ theory if the equations of motion are satisfied. Moreover, if both the equations of motion and the linearized equations of motion hold, then symplectic inner product between $\delta_{1}=\delta$ and $\delta_{2}=\delta_{\xi}$ becomes

$$
\Omega_{\Sigma}\left(\delta A, \delta B, \mathcal{L}_{\xi} A, \mathcal{L}_{\xi} B\right)=\oint_{\partial \Sigma}\left(\delta \mathbf{Q}_{N}[A, B, \xi]-\xi \cdot \boldsymbol{\Theta}(B, \delta A)\right) .
$$

Inserting the explicit expressions for $\mathbf{Q}_{N}[A, B, \xi]$ and $\boldsymbol{\Theta}(B, \delta A)$ one has

$$
\begin{aligned}
\Omega_{\Sigma}\left(\delta A, \delta B, \mathcal{L}_{\xi} A, \mathcal{L}_{\xi} B\right)= & \oint_{\partial \Sigma}\left[\delta\left(\xi \cdot A_{I J}\left(B^{I J}+2 \theta F^{I J}\right)\right)\right. \\
& \left.-\xi \cdot\left(\left(B^{I J}+2 \theta F^{I J}\right) \wedge \delta A_{I J}\right)\right] \\
= & \oint_{\partial \Sigma}\left[\left(\xi \cdot A_{I J}\right) \delta B^{I J}-\left(\xi \cdot B^{I J}\right) \wedge \delta A_{I J}\right],
\end{aligned}
$$

because $F^{I J}=0$ and $\delta F^{I J}=0$, by hypothesis. Last equation has the same analytical form as the one of the BF theory. Therefore, the inclusion of the second Chern character does not modify the degenerate directions in the case of diffeomorphisms.

\section{CONCLUSIONS AND PERSPECTIVES}

To conclude, we emphasize the role that the Bianchi identities $D F_{I J}=0$ play in fourdimesional BF theories. On the one hand, they are the cause of having the symmetry $\delta_{\chi}$ in the various four-dimensional $\mathrm{BF}$ theories already discussed. On the other hand, in the case of the BF theory with a nonvanishing cosmological constant $\Lambda$ the combination of the Bianchi identities together with the equation of motion $F_{I J}=2 \Lambda * B_{I J}$ "generates" dynamics for the $B^{I J}$ fields in the sense that they imply $D B_{I J}=0$. This fact is the origin of the reducibility of the corresponding first class constraints of the theory in Dirac's canonical analysis. This same phenomenon appears, in essence, in the action [1]

$$
S[A, B, \phi]=\int_{\mathcal{M}} B^{I J} \wedge F_{I J}-\frac{1}{2} \phi_{I J K L} B^{I J} \wedge B^{K L},
$$


with $\phi_{I J K L}=-\phi_{J I K L}=-\phi_{I J L K}=\phi_{K L I J}$ where the combination of the Bianchi identities and the equations of motion $F_{I J}=\phi_{I J K L} B^{K L}$ and $D B^{I J}=0$ generates dynamics for the $\phi_{I J K L}$ fields in the sense that these equations imply $\left(D \phi_{I J K L}\right) \wedge B^{K L}=0$. From the lesson learned from the case of the BF theory plus a cosmological constant $\Lambda$, we would expect that the theory defined by Eq. (75) has also reducibility in the constraints in the context of Dirac's analysis. We consider the present analysis as a first step towards the covariant canonical analysis of BF gravity 21].

\section{Acknowledgements}

Warm thanks to J.D. Vergara for discussions about reducibility of constraints and to R. Cartas-Fuentevilla for discussions about the covariant canonical formalism. This work was supported in part by CONACyT grant no. SEP-2003-C02-43939.

\section{APPENDIX A: REVIEW OF DIRAC'S CANONICAL ANALYSIS FOR FOUR- DIMENSIONAL BF THEORY}

To compare some results of this Appendix with some results of the covariant canonical formalism one must make the changes $\varepsilon_{I J} \longrightarrow-\varepsilon_{I J}$ and $\varepsilon_{a}^{I J} \longrightarrow-\chi_{a}^{I J}$ in this Appendix.

(1) BF theory: By making the $3+1$ decomposition, a straightforward computation shows that the action (2) acquires the form

$$
\begin{aligned}
S\left[A_{a I J}, \widetilde{\Pi}^{a I J}, \lambda_{I J}, \lambda_{a}{ }^{I J}\right]= & \int_{\mathcal{M}} d^{4} x\left[\dot{A}_{a I J} \widetilde{\Pi}^{a I J}-\lambda_{I J} D_{a} \widetilde{\Pi}^{a I J}-\lambda_{a}^{I J}\left(\frac{1}{2} \widetilde{\eta}^{a b c} F_{b c I J}\right)\right] \\
& +\int_{\mathcal{M}} d^{4} x \partial_{a}\left(\lambda_{I J} \widetilde{\Pi}^{a I J}\right),
\end{aligned}
$$

where the phase space variables $\left(A_{a I J}, \widetilde{\Pi}^{b K L}\right)$ and Lagrange multipliers $\lambda_{I J}$ and $\lambda_{a}{ }^{I J}$ are defined in terms of the initial configuration variables as $\widetilde{\Pi}^{a I J}:=\frac{1}{2} \widetilde{\eta}^{a b c} B_{b c}{ }^{I J}, \lambda_{I J}:=-A_{0 I J}$, $\lambda_{a}{ }^{I J}:=-B_{0 a}{ }^{I J}, D_{a} \widetilde{\Pi}^{a I J}:=\partial_{a} \widetilde{\Pi}^{a I J}+A_{a}{ }^{I}{ }_{K} \widetilde{\Pi}^{a K J}+A_{a}{ }^{J}{ }_{K} \widetilde{\Pi}^{a I K}$. If the spacetime $\mathcal{M}$ has the topology $\mathcal{M}=\Sigma \times R$ and $\Sigma$ has no boundary the second integral on the right-hand side of (A1) can be neglected. The lower-case letters $a, b$ are space ones and run from 1 to 3 . Notice that the Lorentz indices $I, J$ are not split holding in this way the full Lorentz group. The variation of Eq. (A1) with respect to the phase space variables yields the equations of 
motion

$$
\begin{aligned}
& \dot{A}_{a I J}=-D_{a} \lambda_{I J}, \\
& \dot{\tilde{\Pi}}^{a I J}=2 \lambda^{[I}{ }_{K} \widetilde{\Pi}^{a K J]}-\widetilde{\eta}^{a b c} D_{b} \lambda_{c}{ }^{I J},
\end{aligned}
$$

with $D_{a} \lambda_{I J}=\partial_{a} \lambda_{I J}-A_{a}{ }^{K}{ }_{I} \lambda_{K J}-A_{a}{ }^{K}{ }_{J} \lambda_{I K}$. The variation with respect to the Lagrange multipliers gives the constraints

$$
\widetilde{\Psi}^{I J}:=D_{a} \widetilde{\Pi}^{a I J} \approx 0, \quad \widetilde{\Psi}_{I J}^{a}:=\frac{1}{2} \widetilde{\eta}^{a b c} F_{b c I J}(A) \approx 0
$$

which are first class. The infinitesimal gauge transformation generated by the Gauss constraint $\widetilde{\Psi}^{I J}$ is

$$
A^{\prime}{ }_{a I J}=A_{a I J}-D_{a} \varepsilon_{I J}, \quad \widetilde{\Pi}^{\prime I J}=\widetilde{\Pi}^{a I J}+\varepsilon^{I M} \widetilde{\Pi}^{a}{ }_{M}{ }^{J}-\varepsilon^{J M} \widetilde{\Pi}^{a}{ }_{M}{ }^{I},
$$

and

$$
A^{\prime}{ }_{a I J}=A_{a I J}, \quad \widetilde{\Pi}^{\prime a I J}=\widetilde{\Pi}^{a I J}-\widetilde{\eta}^{a b c} D_{b} \varepsilon_{c}^{I J},
$$

is the gauge transformation generated by the constraint $\widetilde{\Psi}_{I J}^{a}$. However, even though the constraints $\widetilde{\Psi}^{I J}$ are irreducible the constraints $\widetilde{\Psi}_{I J}^{a}$ are not, i.e., they are reducible. This is so because the Bianchi identities $D F_{I J}=0$ imply the relationship among the $\widetilde{\Psi}_{I J}^{a}$ 's,

$$
\widetilde{\Phi}_{I J}:=D_{a} \widetilde{\Psi}_{I J}^{a}=0
$$

The counting of physical degrees of freedom is as follows. There are $3 \times 6=18$ configuration variables $A_{a I J}$ and $6+[(3 \times 6)-6]=18$ independent first class constraints. Therefore, the system has no local degrees of freedom [12]. Alternatively, the independent number of gauge parameters is $18=6$ (the $\varepsilon_{I J}$ 's $)+12\left(=18-6\right.$ independent gauge parameters from $\left.\varepsilon_{a}^{I J}\right)$.

(2) BF theory plus a cosmological constant: By performing the $3+1$ decomposition the action (59) can be written as

$$
\begin{aligned}
S\left[A_{a I J}, \widetilde{\Pi}^{a I J}, \lambda_{I J}, \lambda_{a}{ }^{I J}\right]= & \int d^{4} x\left[\dot{A}_{a I J} \widetilde{\Pi}^{a I J}-\lambda_{I J} D_{a} \widetilde{\Pi}^{a I J}\right. \\
& \left.-\lambda_{a}{ }^{I J}\left(\frac{1}{2} \widetilde{\eta}^{a b c} F_{b c I J}-\Lambda \varepsilon_{I J K L} \widetilde{\Pi}^{a K L}\right)\right] .
\end{aligned}
$$

The equations of motion are

$$
\begin{aligned}
& \dot{A}_{a I J}=-D_{a} \lambda_{I J}-\Lambda \varepsilon_{I J K L} \lambda_{a}{ }^{K L}, \\
& \dot{\widetilde{\Pi}}^{a I J}=2 \lambda^{[I}{ }_{K} \widetilde{\Pi}^{a K J}-\widetilde{\eta}^{a b c} D_{b} \lambda_{c}{ }^{I J} .
\end{aligned}
$$


The constraints are

$$
\widetilde{\Psi}^{I J}:=D_{a} \widetilde{\Pi}^{a I J}, \quad \widetilde{\Psi}_{I J}^{a}:=\frac{1}{2} \widetilde{\eta}^{a b c} F_{b c I J}-\Lambda \varepsilon_{I J K L} \widetilde{\Pi}^{a K L} .
$$

The evolution of the constraints provides no more constraints. To compute the algebra of constraints it is convenient to smear them

$$
\Psi[u]:=\int d^{3} x u_{I J} \widetilde{\Psi}^{I J}, \quad \Psi[N]:=\int d^{3} x N_{a}^{I J} \widetilde{\Psi}_{I J}^{a}
$$

The constraint algebra is

$$
\{\Psi[u], \Psi[v]\}=\Psi[[u, v]], \quad\{\Psi[u], \Psi[N]\}=\Psi[[u, N]], \quad\{\Psi[N], \Psi[M]\}=0,
$$

with $[u, v]_{I J}:=u_{I}{ }^{M} v_{M J}-u_{J}{ }^{M} v_{M I},[u, N]^{I J}{ }_{a}=u_{K}^{I} N^{K J}{ }_{a}-u^{J}{ }_{K} N^{K I}{ }_{a}$. The infinitesimal gauge transformation generated by the Gauss constraint $\widetilde{\Psi}^{I J}$ is

$$
A^{\prime}{ }_{a I J}=A_{a I J}-D_{a} \varepsilon_{I J}, \quad \widetilde{\Pi}^{a I J}=\widetilde{\Pi}^{a I J}+\varepsilon^{I M} \widetilde{\Pi}^{a}{ }_{M}{ }^{J}-\varepsilon^{J M} \widetilde{\Pi}^{a}{ }_{M}{ }^{I},
$$

and

$$
A_{a I J}^{\prime}=A_{a I J}-\Lambda \varepsilon_{I J K L} \varepsilon^{K L}{ }_{a}, \quad \widetilde{\Pi}^{a I J}=\widetilde{\Pi}^{a I J}-\widetilde{\eta}^{a b c} D_{b} \varepsilon_{c}^{I J},
$$

is the infinitesimal gauge transformation generated by the constraint $\widetilde{\Psi}^{a}{ }_{I J}$. Again, the Bianchi identities imply that the constraints are reducible

$$
D_{a} \widetilde{\Psi}_{I J}^{a}+\Lambda \varepsilon_{I J K L} \widetilde{\Psi}^{K L}=0
$$

Like in pure BF gravity the system has $3 \times 6=18$ configuration variables and $6+[(3 \times 6)-6]=$ 18 independent first class constraints. Therefore, the system has no local degrees of freedom, as expected because the addition of a cosmological constant does not add local degrees of freedom. However, a key difference with respect to the case without cosmological constant $\Lambda$ is that there the constraints $\widetilde{\Psi}^{a}{ }_{I J}$ and $\widetilde{\Psi}^{I J}$ are independent while in the present case they are related through the reducibility equation given in Eq. (A14). Moreover, due to the fact the reducibility equation involves now the Gauss constraints too, there are 18 independent gauge parameters among the 6 of $\varepsilon_{I J}$ 's and the 18 of $\varepsilon_{a}^{I J}$ 's. One can take these independent number of gauge parameters as the 18 of the $\varepsilon_{a}^{I J}$ 's. By doing this, one might say that the local Lorentz transformation is redundant if a cosmological constant is present.

[1] R. Jackiw, Rev. Mod. Phys. 52, 661 (1980). 
[2] M. Montesinos and G.F. Torres del Castillo, Phys. Rev. A 70, 032104 (2004) (and references therein); quant-ph/0407051.

[3] M. Montesinos, Class. Quantum Grav. 18, 1847 (2001); gr-qc/0104068.

[4] R. Cartas-Fuentevilla, J. Math. Phys. 45, 602 (2004).

[5] R. Cartas-Fuentevilla and A. Escalante, in Trends in Mathematical Physics Research, edited by C.V. Benton (Nova Science Publishing, USA, 2004).

[6] E. Witten, Nucl. Phys. B 276, 291 (1986).

[7] C. Crnkovic and E. Witten, in Three Hundred Years of Gravitation, edited by S.W. Hawking and W. Israel (Cambridge University Press, Cambridge, 1987).

[8] J. Lee and R.M. Wald, J. Math. Phys. 31, 725 (1990).

[9] A. Ashtekar, L. Bombelli, and O. Reula, in Mechanics, Analysis, and Geometry: 200 years after Lagrange, edited by M. Francaviglia and D. Holm (North-Holland, Amsterdam, 1990).

[10] M. Mondragón, Formalismo canónico covariante de teorías BF en cuatro dimensiones y sistemas parametrizados con un número finito de grados de libertad, M.Sc. Thesis Cinvestav, Mexico, 2003.

[11] G.T. Horowitz, Commun. Math. Phys. 125, 417 (1989).

[12] M.I. Caicedo and A. Restucia, Phys. Lett. B 307, 77 (1993).

[13] M. Mondragón and M. Montesinos, Int. J. Mod. Phys. A 19, 2473 (2004); gr-qc/0312048.

[14] M. Montesinos, in VI Mexican School on Gravitation and Mathematical Physics, edited by M. Alcubierre, J.L. Cervantes-Cota, and M. Montesinos, Journal of Physics: Conference Series 24 (Institute of Physics Publishing, Bristol and Philadelphia, 2005); gr-qc/0602072.

[15] M. Montesinos, Class. Quantum Grav. (2006) (to appear).

[16] J.C. Baez, Lect. Notes Phys. 543, 25 (2000).

[17] M. Montesinos, Class. Quantum Grav. 20, 3569 (2003).

[18] V. Iyer and R.M. Wald, Phys. Rev. D 52, 4430 (1995).

[19] G.A. Burnett and R.M. Wald, Proc. R. Soc. London A 430, 57 (1990).

[20] R. De Pietri and L. Freidel, Class. Quantum Grav. 16, 2187 (1999).

[21] R. Capovilla, M. Montesinos, V. A. Prieto, and E. Rojas, Class. Quantum Grav. 18, L49 (2001); gr-qc/0102073 